\title{
Rapidity divergences and valid definitions of parton densities
}

\section{John COLLINS*}

Physics Department, Pennsylvania State University, USA

E-mail: collins@phys.psu . edu

Rapidity divergences occur when parton densities in a gauge theory are defined in the most natural way, as expectation values of partonic number operators in light-front quantization. I review these and other related divergences, and show how the definitions of parton densities can be modified to remove the divergences. A modified definition is not only essential for many phenomenological applications of QCD, but also concerns the treatment of parton densities in non-perturbative approaches. The necessity of modifications in the definition of a parton density also entails corrections in the formulation of light-front quantization for gauge theories.

LIGHT CONE 2008 Relativistic Nuclear and Particle Physics

July 7-11, 2008

Mulhouse, France

\footnotetext{
* Speaker.
} 


\section{Introduction}

Parton densities are central to much phenomenology of scattering in QCD. Hard-scattering factorization theorems represent cross sections as convolutions of perturbatively calculable factors and of parton densities (and related quantities like fragmentation functions). Predictions are made on the basis of perturbative calculations because of the universality of parton densities between different reactions.

Natural candidate definitions of parton densities are obtained from elementary treatments of the parton model, and they can be formulated as expectation values of number operators in lightfront quantization. Unfortunately, when applied in a gauge theory like QCD, these definitions suffer from divergences where the rapidities of some gluons goes to infinity.

This talk reviews how rapidity divergences and certain other divergences arise. It summarizes their impact on correct definitions of parton densities, on phenomenology, and on fundamental issues in light-front quantization. Naturally, a valid derivation of a factorization property requires a valid definition of parton densities that is matched to the factorization property. Moreover, to allow non-perturbative methods in QCD to be used to estimate parton densities, operator definitions of parton densities are needed that can be taken literally.

\section{Parton densities from parton model}

In view of the many complications in full QCD, we first recall how parton densities arise in the parton model. This leads to a natural definition of a parton density, thereby giving a convenient conceptual landmark. The results in QCD can be regarded, not as overthrowing the parton model but as modifying and distorting it, while preserving much of an overall intuitive framework.

We consider DIS, $e p \rightarrow e X$, and use light-front coordinates: $k^{ \pm}=\left(k^{0} \pm k^{z}\right) / \sqrt{2}$. We work in the Breit frame, where the momenta of the target and the virtual photon are $p^{\mu}=\left(p^{+}, M^{2} /\left(2 p^{+}\right), \mathbf{0}_{T}\right)$ and $q^{\mu}=\left(-x p^{+}, Q^{2} /\left(2 x p^{+}\right), \mathbf{0}_{T}\right)$. In the parton model, valid in certain model field theories, we assume that, to leading power in mass $/ Q$, there is dominance by handbag diagrams

$$
F_{2} \simeq \int \frac{\mathrm{d}^{4} k}{(2 \pi)^{4}}
$$

with the virtualities and transverse momentum of the quark lines being limited.

This leads to well-known scaling formulae for structure functions, such as $F_{2}=\sum_{j} e_{j}^{2} x f_{j}(x)$. Here $f_{j}$ is the density of a quark of flavor $j$ defined graphically by

$$
f_{j}(x) \stackrel{\text { parton model }}{=} \operatorname{Tr} \frac{\gamma^{+}}{2} \int \frac{\mathrm{d} k^{-} \mathrm{d}^{2} \mathbf{k}_{T}}{(2 \pi)^{4}}
$$

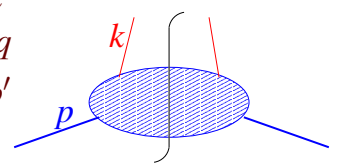

with $k^{+}=x p^{+}$, which relates the momentum of the internal quark to the experimentally measurable quantity $x$. The corresponding operator formula is

$$
f_{j}(x) \stackrel{\text { parton model }}{=} \int \frac{\mathrm{d} w^{-}}{2 \pi} e^{-i x P^{+} w^{-}}\left\langle P\left|\bar{\psi}_{j}\left(0, w^{-}, \mathbf{0}_{T}\right) \frac{\gamma^{+}}{2} \psi_{j}(0)\right| P\right\rangle_{\mathrm{c}},
$$


where $\psi_{j}\left(w^{+}, w^{-}, \mathbf{w}_{T}\right)$ is the quark field, and the subscript "c" denotes that only connected graphs are to be included. The two fields in Eq. (2.3) have a light-like separation, because of the integral over all $k^{-}$and $\mathbf{k}_{T}$ in Eq. (2.2).

Some other processes, e.g., semi-inclusive DIS, are sensitive to parton transverse momentum. For these, we use an unintegrated parton density defined by removing the integral over $\mathbf{k}_{T}$, to give

$$
f_{j}\left(x, \mathbf{k}_{T}\right) \stackrel{\text { parton model }}{=} \int \frac{\mathrm{d} w^{-} \mathrm{d}^{2} \mathbf{w}_{T}}{(2 \pi)^{3}} e^{-i x P^{+} w^{-}+i \mathbf{k}_{T} \cdot \mathbf{w}_{T}}\left\langle P\left|\bar{\psi}_{j}\left(0, w^{-}, \mathbf{w}_{T}\right) \frac{\gamma^{+}}{2} \psi_{j}(0)\right| P\right\rangle_{\mathrm{c}} .
$$

An interpretation is made by using light-front quantization. Expanding the fields in annihilation and creation operators gives

$$
f_{j}\left(x, \mathbf{k}_{T}\right) \stackrel{\text { parton model }}{=} \sum_{\lambda} \frac{\left\langle p\left|b_{k, \lambda, j}^{\dagger} b_{k, \lambda, j}\right| p\right\rangle-\text { vacuum expectation value }}{\langle p \mid p\rangle} \times \frac{1}{2 x(2 \pi)^{3}},
$$

where $b_{k, \lambda, j}$ is the annihilation operator for a quark of flavor $j$, helicity $\lambda$, plus-momentum $x p^{+}$and transverse momentum $\mathbf{k}_{T}$. To the extent that the parton model is exactly correct, a parton density is therefore the number density of partons of the given momentum and flavor.

\section{Wilson lines in parton densities}

\subsection{Light-cone gauge}

To apply light-front quantization in a gauge theory, it is natural to use the light-cone gauge $A^{+}=0$. For partons collinear to the target, this gauge leads to a treatment that is the same as in the parton model. So it is very natural to try to continue to use Eq. (2.4), or equivalently (2.5). However, such a definition gives problems from kinematic regions other than the collinear-to-target region.

We formulate the gauge condition covariantly as $n \cdot A=0$, where $n^{\mu}=\delta_{-}^{\mu}$. Then the gluon propagator has a singularity at $k \cdot n=0$ :

$$
\frac{i}{k^{2}}\left(-g^{\mu v}+\frac{k^{\mu} n^{v}+n^{\mu} k^{v}}{k \cdot n}\right)
$$

Now, in proving factorization, certain contour deformations are applied to avoid a rescattering region. Some relevant graphs are illustrated in Fig. 1(a). The contour deformation is away from $k \cdot n \simeq 0$, and is obstructed by the gauge singularity in the light-cone-gauge propagator Eq. (3.1).

To avoid this problem, we use Feynman gauge. But the price is that we have extra regions to consider, notably where the gluons in Fig. 1(a) are collinear to the target, a situation that is power suppressed in light-cone gauge.

\subsection{Wilson lines in integrated parton densities}

The first part of the solution is to make the definition of the parton densities gauge-invariant, by inserting a Wilson-line operator between the quark and anti-quark fields. A Wilson line is a path-ordered exponential of the gluon field taken along some path joining the two fields. The 


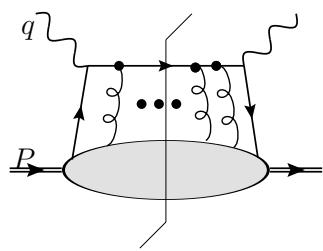

(a)

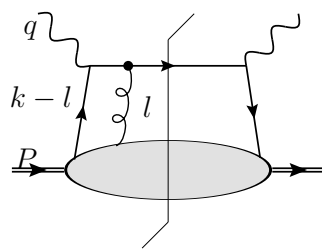

(b)

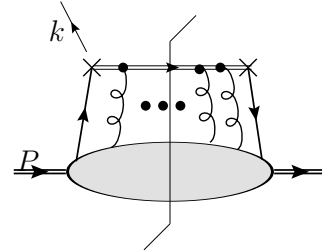

(c)

Figure 1: (a) Final-state gluon exchanges added to a handbag diagram are of leading power. (b) One-gluon exchange, with momentum labeling. (c) Parton density with Wilson line.

key question is which is the appropriate path if we are to obtain a parton density that allows the derivation of a valid factorization property for the cross section.

In the case of an integrated density, (2.3), the fields are separated along exactly the minus direction. Taking the Wilson line along the path joining the two fields is appropriate [1], so that we insert into the matrix element a factor

$$
W\left(w^{-}, 0\right)=P \exp \left(-i g_{0} \int_{0}^{w^{-}} \mathrm{d} y^{-} A_{(0) \alpha}^{+}\left(0, y^{-}, \mathbf{0}_{T}\right) t_{\alpha}\right)
$$

which is equal to unity in $A^{+}=0$ gauge. Graphs like Fig. 1(a) with gluon exchanges between the final-state quark and everything else, lead to graphs like Fig. 1(c) for the parton density, with the double line indicating the Feynman rules for the Wilson line: The definition matches the proof.

Supplemented by renormalization of the UV divergences in the parton density, this definition is satisfactory for integrated parton densities, as far as is known. These are the standard parton densities that are used in much phenomenology.

\subsection{Meaning of Wilson line}

The Wilson line arises from an approximation to the momentum that flows from a gluon onto a final-state quark. For example, for exchange of one gluon of momentum $l$, Fig. 1(b), we have

$$
\frac{1}{(q+k-l)^{2}-m^{2}+i \varepsilon} \simeq \frac{1}{-2(q+k) \cdot l+i \varepsilon} \simeq \frac{1}{-2 q^{-} l^{+}+i \varepsilon} \text {. }
$$

The approximation is valid for a gluon collinear to the target, and the previously mentioned contour deformation is needed to avoid the region where $l^{+}$gets small with $\mathbf{l}_{T}$ fixed.

We interpret this formula by regarding the outgoing quark as having a diffraction pattern arising from quark emission from different points in the target, Fig. 2. The quark is highly time-dilated in the rest frame of the target, and is therefore almost undeflected while it remains inside the target. The Wilson line essentially gives the quantum-mechanical phase acquired by the quark as it climbs out of gluon field of the target. The Sivers function, a transverse-spin-dependent unintegrated parton density that we will hear about elsewhere in this workshop, occurs because in a spinning proton, the gluon field rotates and gives different phases on opposite sides of the proton, just as for optical diffraction from a rotating transparent ring. 


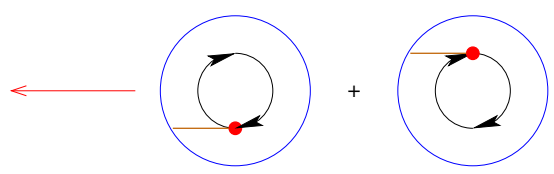

Figure 2: A quark is knocked out of proton by a virtual photon, and acquires acquires a phase accumulated along its path.

\section{Unintegrated pdf and rapidity divergences}

For an unintegrated density (2.4), the parton fields are no longer at a light-like separation, but the derivation of factorization still requires us to use manipulations like those in Eq. (3.3). Thus the Wilson line does not go along the line joining the quark and anti-quark; instead, it goes out to infinity from one field in an approximately light-like direction, makes a transverse jog, and then comes back to the other field. Thus the interpretation still applies that the Wilson line gives the phase acquired by a fast-moving quark going through the gluon field of the target. That the Wilson line goes to future infinity, not to past infinity, is determined by the sign of the $i \varepsilon$ in (3.3).

With exactly light-like Wilson line, there are divergences at $l_{\text {gluon }}^{+}=0$. For example, for a graph with one exchanged real or virtual gluon, we have

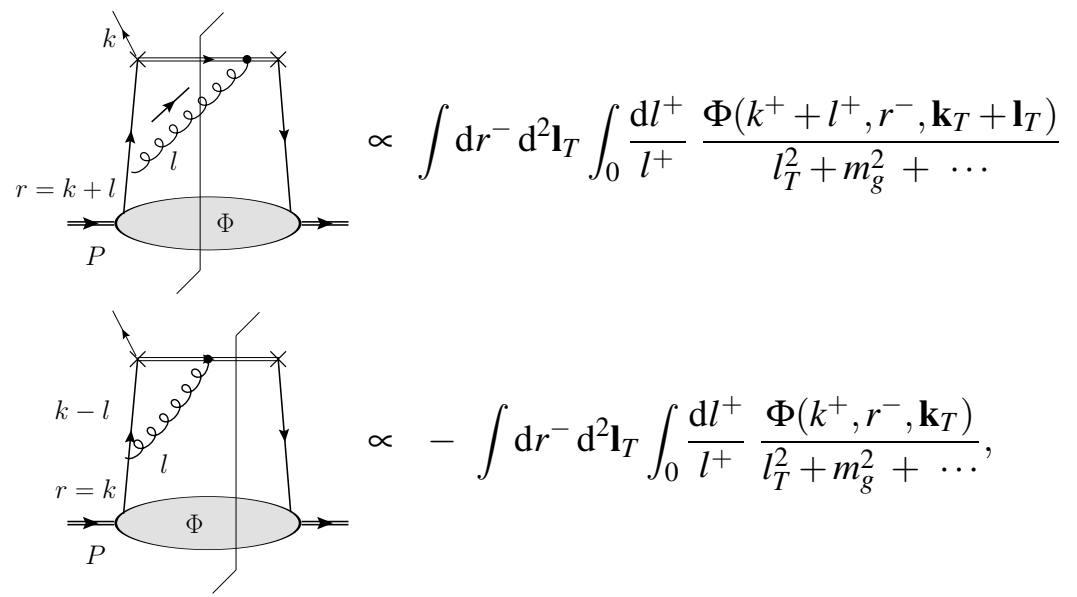

where the dots "..." indicate terms that vanish at $l^{+}=0$ and that therefore do not contribute to the divergence. The two graphs differ only by placement of the final-state cut. If we were to integrate over all external transverse momenta $\mathbf{k}_{T}$, to get a contribution to the integrated quark density, the divergence would cancel. But in the unintegrated density, the divergence is uncanceled, and does not cancel against other graphs. The same divergence occurs in light-front wave functions - see, for example, Eq. (29) of [2].

We included a non-zero gluon mass in Eq. (4.1), to demonstrate clearly that the divergence is not an infra-red divergence, even though one component of gluon momentum goes to zero. Equally it cannot be regulated by changing the dimension of space-time.

The divergence, in fact, arises from an integral over gluon rapidity, defined by

$$
y \equiv \frac{1}{2} \ln \frac{l^{+}}{l^{-}}=\ln \frac{l^{+}}{\sqrt{\left(l^{2}+l_{T}^{2}\right) / 2}} .
$$




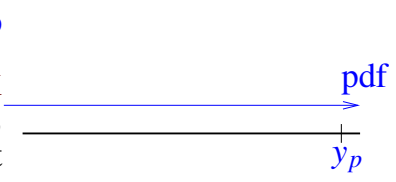

(a)

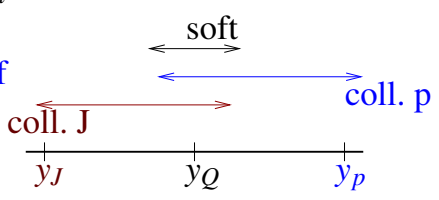

(b)

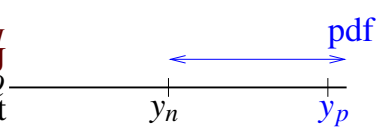

(c)

Figure 3: Rapidity ranges: (a) for parton density with light-like Wilson line; (b) for original integral; (c) for parton density with Wilson line of rapidity $y_{n}$.

Changing variable from $l^{+}$to $y$ at fixed $\mathbf{l}_{T}$ gives an approximately uniform integrand as a function of $y$, between kinematic limits. The upper limit is the proton rapidity, but - Fig. 3(a) - the lower limit, given by the Wilson line, is $-\infty$.

This misrepresents the actual physics, Fig. 3(b). Only the target-collinear range, approximately between the target and the virtual photon, belongs in the parton density. Much lower rapidities belong with the jet, while a central range should be associated with a soft factor. Naturally in a factorization theorem, it is necessary to compensate double counting of the overlap regions.

\subsection{Modified parton density with cut off on rapidity}

We are therefore required to modify the definition of the parton density to provide some kind of cut off on gluon rapidity.

One obvious solution is to make the direction $n$ of the Wilson line non-light-like. This is essentially the solution of Collins and Soper [1,3], except that they used a non-light-like axial gauge $n \cdot A=0$. As we have already mentioned, such gauges cause problems in properly deriving factorization, so the Wilson line solution is preferred. The definition of a parton density now has the Wilson line going to $+\infty$ in direction $n$, making a jog at infinity, and coming back:

$$
\begin{aligned}
f_{j}\left(x, \mathbf{k}_{T} ; y_{n}\right)=\int \frac{\mathrm{d} w^{-} \mathrm{d}^{2} \mathbf{w}_{T}}{(2 \pi)^{3}} e^{-i x P^{+} w^{-}+i \mathbf{k}_{T} \cdot \mathbf{w}_{T}} \\
\qquad p, s \mid \bar{\psi}\left(0, w^{-}, \mathbf{w}_{T}\right) W(w \text { to } \infty ; n)^{\dagger} \frac{\gamma^{+}}{2} W(\text { at } \infty) W(0 \text { to } \infty ; n) \psi(0)|p, s\rangle .
\end{aligned}
$$

Other variations on the same idea are possible, e.g., [4], but there must always be present an extra parameter $y_{n}$ that limits the gluon rapidity: Fig. 3(c).

Collins, Soper and Sterman (CSS) $[1,3,5]$ restored the predictive power of the theory by an equation for the $y_{n}$ dependence. The kernel of the equation involves a perturbatively calculable function $G$, a universal non-perturbative function $K\left(q_{T}\right)$, and a perturbatively calculable renormalization-group function $\gamma_{K}\left(\alpha_{s}\right)$ for $K$ and $G$.

The CSS formalism not only determines a resummation of the large logarithms of $Q / q_{T}$ that arise in collinear factorization, but systematically treats the effects of the non-perturbative region of small transverse momentum for the partons.

\subsection{Implications}

An immediate consequence of the CSS method is the energy dependence of the transversemomentum distribution of the Drell-Yan cross section. Fits to the non-perturbative parts of the factorization formula are made, in principle from a subset of the data, and predictions then made 


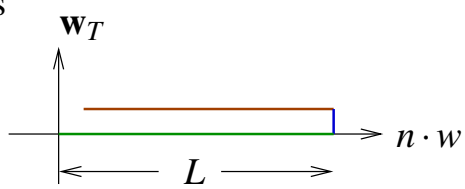

Figure 4: Wilson line in unintegrated parton density, viewed from side. The limit $L \rightarrow \infty$ should be taken.

for all energies, e.g., [6]. Extending the formalism to the gluon density allows the transverse momentum of the Higgs boson to be predicted, e.g., [7]. Similar methods apply to transverse momentum distributions in other processes, like semi-inclusive DIS, and almost-back-to-back hadron production in $e^{+} e^{-}$annihilation.

Close examination of the arguments for the direction of the Wilson lines shows that in the Drell-Yan process, the Wilson lines must be point to the past instead of the future [8]. Time-reversal symmetry of QCD shows that for most parton densities, the change in the direction of Wilson line compared with DIS has no effect on the numerical value of the parton density. But certain quantities involving transverse spin change sign [9] between DIS and Drell-Yan. These include the Sivers function, which governs the transverse-momentum density of partons in a transversely polarized nucleon, and the Boer-Mulders function, which governs the transverse polarization of quarks in a unpolarized hadron.

\section{Wilson line self energy?}

Further complications arise because there is a segment of the Wilson line that can be regarded as a color dipole extending all the way to infinity - Fig. 4. Certain topologies of graph are required in the parton density, but do not arise in the derivation of factorization, e.g., in obtaining Fig. 1(c) from Fig. 1(a).

One complication, found by Belitsky, Ji, and Yuan [10], is that definition (4.3) entails graphs like Fig. 5 that have gluons connecting the lower part of the graph to the segment of the Wilson line that makes the transverse jog at infinity. These graphs vanish in Feynman gauge, so they do not affect the proof of factorization. But they do appear in the $n \cdot A=0$ gauge, to give gaugeindependent parton densities. This resolves the problem that otherwise the Sivers function is zero in axial gauge.

A second complication, not apparently recognized before, gives rise to extra divergences. To solve it, I propose here a further redefinition of the unintegrated quark density to cancel the undesired contributions. While this change affects the actual operator definition, it leaves the CSS evolution equations unaltered. Thus it leaves unaltered the phenomenology described in the previous section, which uses the form of the evolution equation but not the full implementation of the operator definition.

The divergences were found by Diehl (private communication) using the Collins-Soper definition in $n \cdot A=0$ gauge. In the Feynman gauge, the divergence arises from Wilson line self energies. The one-gluon case, Fig. 6, gives a linear divergence, which in coordinate space is proportional to the length $L$ of the Wilson line, in the limit $L \rightarrow \infty$. This graph does not correspond to any treated in the factorization proof. 


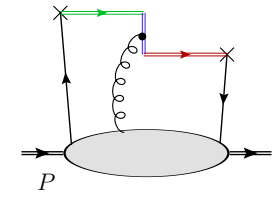

Figure 5: Gluons joining to the "jog-at-infinity" part of the Wilson line, color-coded as in Fig. 4.

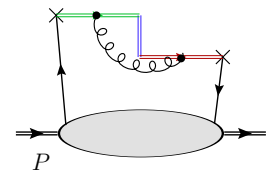

Figure 6: Self-energy graph for Wilson line.

A natural expectation, in need of a full proof, is that to all orders, and also beyond perturbation theory, the infinitely long dipole will have $L$-dependence like that of a Wilson loop, i.e., there is an exponentiation, giving a factor of the form $e^{-L V\left(w_{T}\right)}$ in coordinate space, where $V\left(w_{T}\right)$ depends on the separation $w_{T}$ between the two parts of the Wilson line. This divergence should be exactly the same as in a Wilson loop of size $L \times w_{T}$.

It is also necessary to cancel graphs where gluons connect the long sections of the Wilson line to the jog at infinity, including the associated UV divergences, since none of these graphs appear in the factorization proof. Canceling these graphs avoids the problems discussed in [11].

I therefore propose to redefine the unintegrated parton density as follows:

$$
f_{j}\left(x, \mathbf{k}_{T} ; y_{n}\right)=\int \frac{\mathrm{d} w^{-} \mathrm{d}^{2} \mathbf{w}_{T}}{(2 \pi)^{3}} e^{-i x P^{+} w^{-}+i \mathbf{k}_{T} \cdot \mathbf{w}_{T}} \lim _{L \rightarrow \infty} \frac{\text { Matrix element in }(4.3)(L)}{\sqrt{ }} .
$$

The numerator is just as in Eq. (4.3), but with the Wilson line going out to $n \cdot y=L$ instead of $\infty$. In the denominator is a Wilson loop. Since it has two transverse segments, whereas the numerator has only one, we take the square root of the Wilson loop. Hence, to keep the correct $e^{-L V\left(w_{T}\right)}$ divergence factor, the Wilson loop must be given a length $2 L$, when the numerator has a Wilson line has length $L$. Finally the limit $L \rightarrow \infty$ is taken.

We should now have a satisfactory definition, to be taken literally in any kind of calculation (perturbative or non-perturbative). Various further refinements are possible, e.g., to absorb soft factors in the CSS factorization formula, but these can be made in terms of the quantity just defined.

\section{Summary and outlook}

An elementary treatment of DIS in a field theory where the parton model is valid leads to a definition of a parton density that can be literally interpreted as an expectation value of a parton number operator in the sense of light-front quantization.

In QCD the definition must be modified by the insertion of a Wilson line. A natural definition is to use a light-like Wilson line. But in an unintegrated density, this gives rapidity divergences, which must be cut-off or avoided somehow, for example by a non-light-like Wilson line. The rapidity cutoff $y_{n}$ is tied to the physics of the cross section, and there is an equation for the evolution with respect to $y_{n}$.

A new result is that further divergences arise from the integral to spatial infinity of the dipolar Wilson line. These can be canceled by a suitable Wilson loop factor, without affecting the standard CSS phenomenology. Further work is needed to test whether my conjectures in this area are correct. 
Note added after presentation of the talk: The same divergences infect light-front wave functions. This presumably necessitates some changes or distortions in standard formulations of QCD quantized on the light-front, if the formalism is to be taken literally.

\section{References}

[1] J.C. Collins and D.E. Soper, Nucl. Phys. B 194, 445 (1982).

[2] S.J. Brodsky, D.S. Hwang, B.Q. Ma and I. Schmidt, Nucl. Phys. B 593, 311 (2001) [arXiv:hep-th/0003082].

[3] J.C. Collins and D.E. Soper, Nucl. Phys. B 193, 381 (1981) [Erratum-ibid. B 213, 545 (1983)].

[4] X. Ji, J. Ma and F. Yuan, Phys. Rev. D 71, 034005 (2005) [arXiv:hep-ph/0404183].

[5] J.C. Collins, D.E. Soper and G. Sterman, Nucl. Phys. B 250, 199 (1985).

[6] F. Landry, R. Brock, P.M. Nadolsky and C.P. Yuan, Phys. Rev. D 67, 073016 (2003) [arXiv:hep-ph/0212159].

[7] E.L. Berger and J. Qiu, Phys. Rev. D 67, 034026 (2003) [arXiv:hep-ph/0210135].

[8] J.C. Collins and A. Metz, Phys. Rev. Lett. 93, 252001 (2004) [arXiv:hep-ph/0408249].

[9] J.C. Collins, Phys. Lett. B 536, 43 (2002) [arXiv:hep-ph/0204004].

[10] A.V. Belitsky, X. Ji and F. Yuan, Nucl. Phys. B 656, 165 (2003) [arXiv:hep-ph/0208038].

[11] I.O. Cherednikov and N.G. Stefanis, Phys. Rev. D 77, 094001 (2008) [arXiv:0710.1955 [hep-ph]]. 